\title{
Majorana fermions debut
}

Signatures of Majorana fermions in hybrid superconductor-semiconductor nanowire devices, V. Mourik, K. Zuo, S.M. Frolov, S.R. Plissard, E.P.A.M. Bakkers, and L.P. Kouwenhoven, Science, 12 April 2012 [arXiv: 1204.2792].

Recommended with a commentary by Carlo Beenakker, Leiden University

The experiment reported by Leo Kouwenhoven and his team from Delft University can be described in one sentence: Take a semiconductor nanowire $(\mathrm{InSb})$, place it in a parallel magnetic field, connect one end to a grounded superconductor and the other end via a tunnel barrier to a voltage source, then measure the current. A plot of the differential conductance $G(V)=$ $d I / d V$ has familiar and unfamiliar features. A familiar feature is the large peak that appears when $\pm e V$ equals the superconducting gap $\Delta \approx 250 \mu \mathrm{eV}$. The unfamiliar feature, that is generating much excitement, is a small peak (height $\approx 0.1 e^{2} / h$ ) at zero voltage. Why the excitement?

Experiments of this type typically produce peaks when $\mathrm{eV}$ equals the energy $E$ of a bound state inside the gap. A peak at $V=0$ indicates a bound state right in the middle of the gap. Nothing particulary interesting, unless the state is nondegenerate. The particle/antiparticle symmetry relation for creation/annihilation operators, $\gamma(E)=\gamma^{\dagger}(-E)$, implies that a nondegenerate state at $E=0$ has identical particle and antiparticle, $\gamma=\gamma^{\dagger}$. This defines a Majorana fermion.

The absence of degeneracies is crucial: for example, if the midgap state would be spin-up/down degenerate one would have $\gamma_{\uparrow}=\gamma_{\downarrow}^{\dagger}$, so distinct particle and antiparticle. The purpose of the magnetic field in the experiment is to remove degeneracies, and it seems to work: The peak in $G(V)$ is pinned to $V=0$ upon variation of the magnetic field (in the range 100-400 $\mathrm{mT}$ ) or Fermi energy (by up to $1 \mathrm{meV}$ ). This indicates that the midgap state cannot move up or down in energy without breaking the required $\pm E$ symmetry. If the midgap state would have been degenerate, it could have split into a pair of levels at $\pm E$. Indeed, pairs of peaks are observed at higher magnetic fields that cross through $V=0$ in a $\pm V$ symmetric way, indicating a degenerate pair of levels, but the midgap state at lower fields stays fixed at zero voltage.

The midgap state disappears rather abruptly if the magnetic field is reduced below $100 \mathrm{mT}$. It also disappears if the field is oriented perpendicular, rather than parallel, to the wire, in the direction of the spin-orbit coupling field. Finally, there is no peak at zero voltage if the superconductor is replaced by a normal-metal electrode. These are the three necessary ingredients - magnetic field, spin-orbit coupling, and superconductivity — for the emergence of a Majorana fermion at the end points of a nanowire. Indeed, 
the experiment was designed according to the specifications of two theoretical groups (Lutchyn, Sau \& Das Sarma; Oreg, Refael \& Von Oppen), being among the first of many Majorana proposals to be realized in the laboratory. (See arXiv:1112.1950 and arXiv:1202.1293 for two recent reviews.)

Interpreting the experiment along these lines, the appearance of a midgap state signals the closing and reopening of the excitation gap. The magnetic field closes the gap by aligning the electron spins and breaking up the (spinup/spin-down) Cooper pairs injected by the superconductor. The spin-orbit coupling field (pointing in a different direction) can counteract this effect by flipping the spin, reopening the excitation gap. The closing and reopening of the gap leaves behind a defect, in the form of a bound state at the end points of the wire. In a normal metal these are the familiar Shockley states. In a superconductor the particle-antiparticle symmetry pins the Shockley states to the middle of the gap, where they are Majorana fermions.

The Majorana interpretation is accepted by many in the community, mainly because it is the simplest and most obvious explanation, requiring only single-particle physics. Majorana fermions have other experimental signatures, that would confirm the identification. One signature might be observed in the same nanowire geometry, upon replacement of the tunnel barrier by a ballistic point contact. The linear-response conductance $G(0)$ is then predicted to be quantized at $n G_{0}$ without a Majorana and $\left(n+\frac{1}{2}\right) G_{0}$ with a Majorana, where $G_{0}=4 e^{2} / h$ is the Andreev conductance quantum. The half-integer conductance quantization arises because the Majorana fermion is in essence only half a conventional fermion.

Other Majorana signatures could be searched for if the nanowire connects two superconductors, forming a Josephson junction. A supercurrent can flow through a Josephson junction in the absence of any applied voltage, producing a peak in $d I / d V$ at $V=0$ even without Majoranas. This complicates the Majorana interpretation of zero-voltage peaks in a Josephson junction, reported in arXiv:1204.4130, but there is an altogether different signature: The pair of midgap states at the two superconducting interfaces forms a channel for the transport of single electrons through the Josephson junction, in addition to the usual $2 e$ Cooper pairs. One would therefore expect an enhancement of the $h / e$ Shapiro step when the Majorana fermions emerge. Puzzlingly enough, the opposite effect has been reported in arXiv:1204.4212 (a suppression of the $h / 2 e$ Shapiro step).

The "holy grail" in this topic is the demonstration of the non-Abelian braiding statistics of the midgap states. Since $N$ Majorana fermions have a $2^{N / 2}$-fold degenerate ground state, a sequence of pairwise exchanges (braiding) amounts to a non-commuting unitary-matrix multiplication rather than the usual multiplication of phase factors. Nanowires can be grown to form 
T-junctions and X-junctions, through which one can imagine exchanging the Majoranas. There is no need to physically move the end points of the wires, by varying the coupling of neighboring Majoranas one can implement the same sequence of unitary-matrix multiplications. All of this may, on the long-term, have implications for topological quantum computing, but on the short-term there is exciting fundamental physics to be discovered. 